\title{
New Insights into Cartilage Tissue Engineering: Improvement of Tissue-Scaffold Integration to Enhance Cartilage Regeneration
}

\author{
Sahar Jelodari $\left(\mathbb{D},{ }^{1,2}\right.$ Amin Ebrahimi Sadrabadi $\left(\mathbb{D},{ }^{3}\right.$ Fatemeh Zarei $(\mathbb{D})^{3}$ \\ Shahrbanoo Jahangir ${ }^{(D)},{ }^{4}$ Mahmoud Azami $\left(\mathbb{D},{ }^{1}\right.$ Mohsen Sheykhhasan ${ }^{(D)}, 5,6$ \\ and Samaneh Hosseini $\mathbb{D D}^{2,3}$ \\ ${ }^{1}$ Department of Tissue Engineering, School of Advanced Technologies in Medicine, Tehran University of Medical Sciences, \\ Tehran, Iran \\ ${ }^{2}$ Department of Cell Engineering, Cell Science Research Center, Royan Institute for Stem Cell Biology and Technology, ACECR, \\ Tehran, Iran \\ ${ }^{3}$ Department of Stem Cells and Developmental Biology, Cell Science Research Center, Royan Institute for Stem Cell Biology \\ and Technology, ACECR, Tehran, Iran \\ ${ }^{4}$ AO Research Institute Davos, Davos, Switzerland \\ ${ }^{5}$ Research Center for Molecular Medicine, Hamadan University of Medical Sciences, Hamadan, Iran \\ ${ }^{6}$ Department of Mesenchymal Stem Cells, Academic Center for Education, Culture and Research (ACECR), Qom Branch, Qom, Iran
}

Correspondence should be addressed to Mohsen Sheykhhasan; mohsen.sh2009@gmail.com

and Samaneh Hosseini; hosseini.samaneh@royaninstitute.org

Received 25 July 2021; Revised 20 December 2021; Accepted 29 December 2021; Published 25 January 2022

Academic Editor: You-Shui Gao

Copyright (c) 2022 Sahar Jelodari et al. This is an open access article distributed under the Creative Commons Attribution License, which permits unrestricted use, distribution, and reproduction in any medium, provided the original work is properly cited.

Distinctive characteristics of articular cartilage such as avascularity and low chondrocyte conversion rate present numerous challenges for orthopedists. Tissue engineering is a novel approach that ameliorates the regeneration process by exploiting the potential of cells, biodegradable materials, and growth factors. However, problems exist with the use of tissue-engineered construct, the most important of which is scaffold-cartilage integration. Recently, many attempts have been made to address this challenge via manipulation of cellular, material, and biomolecular composition of engineered tissue. Hence, in this review, we highlight strategies that facilitate cartilage-scaffold integration. Recent advances in where efficient integration between a scaffold and native cartilage could be achieved are emphasized, in addition to the positive aspects and remaining problems that will drive future research.

\section{Background}

Cartilage is a specialized connective tissue with an extracellular matrix (ECM) that is rich in glycosaminoglycans (GAGs) and proteoglycans (PGs). The ECM structure of articular cartilage enables it to reduce pressures and absorb shocks inflicted on the subchondral bone and provides a slippery surface which facilitate bone movement $[1,2]$. Articular cartilage exhibits very low intrinsic healing capacity due to its avascular nature and scarcity of cells [3]; therefore, its injury or damage results in pain and loss of mobility in patients, and the need for medical intervention is its inevitable conse- quence. Despite numerous attempts in pharmaceutical and surgical therapies, they are unable to successfully restore damaged cartilage [4-9]. Cartilage tissue engineering (CTE) has the potential to enhance healing by embedding relevant cells like articular chondrocytes or mesenchymal stem cells (MSCs) and growth factors (GFs) into scaffolds in order to support cell growth and proliferation (Figure 1) [10-12]. Natural (collagen, gelatin, fibrin, silk fibroin, alginate, hyaluronan, chondroitin sulfate, agarose, and chitosan) and synthetic (polyethylene glycol, poly (lactide-co-glycolic) acid [PLGA], and polycaprolactone [PCL]) biomaterials have been used in CTE [13-15]. However, integration between 


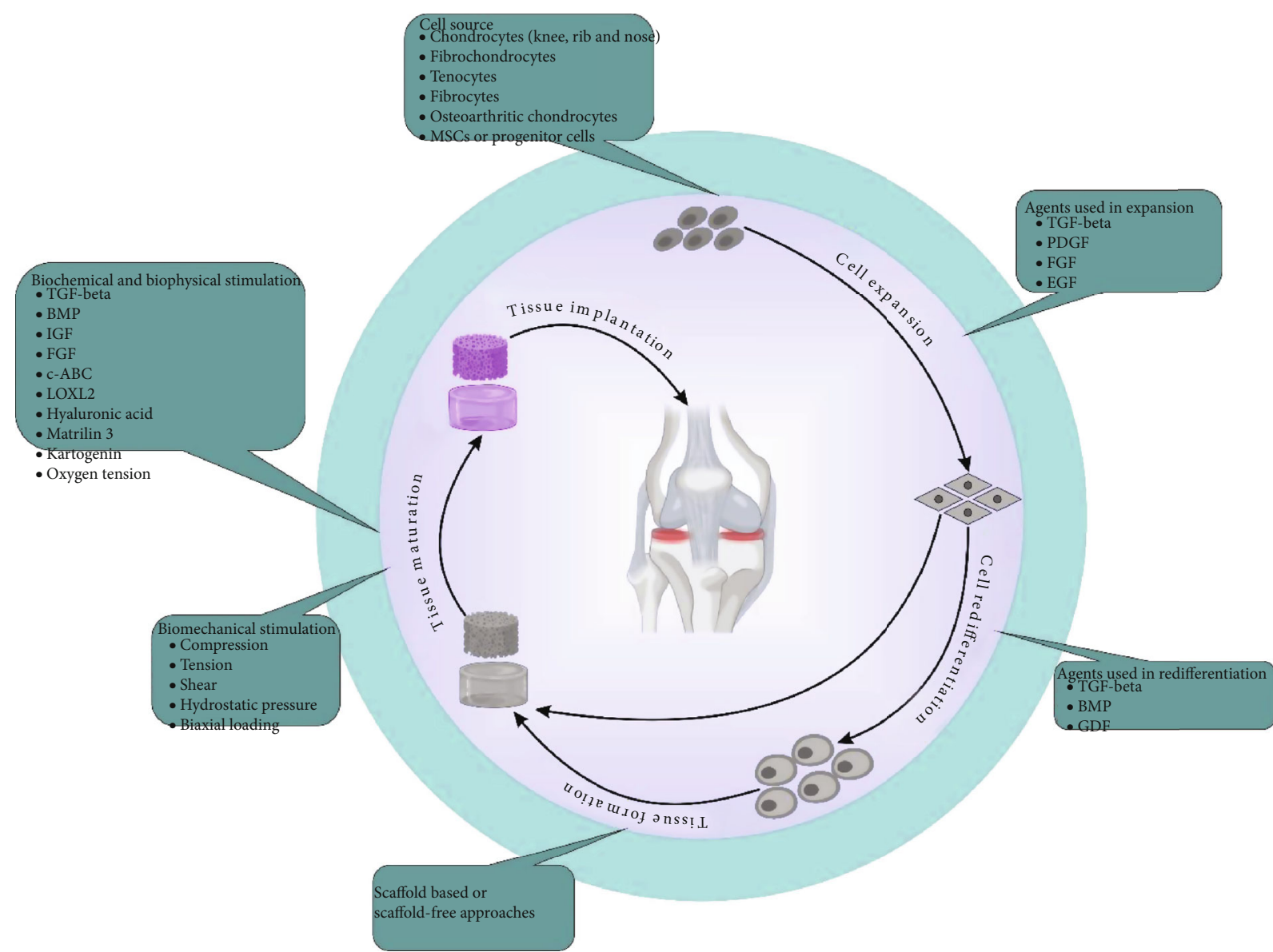

FIGURE 1: Tissue engineering of cartilaginous tissue. Factors and components like cells, various biochemical, biophysical, and biomechanical stimulations and scaffolds contribute to the development of a well-designed tissue-engineered construct.

native cartilage and neoformed tissue remains challenging. Lack of integration can be counterintuitive and lead to further cartilage degeneration [16]. Although suturing or applying adhesives are the current approaches for achieving certain extent of tissue integrity, either of them has its own pitfalls [17]. In this review, we discuss chemical composition, porosity, and load-bearing capacity of biomaterials as properties that can impact scaffold-cartilage integration. Scaffold-free cartilage integration and targeted cell delivery are mentioned as cell-based approaches to repair cartilage defects. Relevant biomolecules in the realm of cartilage regeneration that contribute to seamless integration are discussed. We also explore bioprinting and recruitment of extracellular vesicles as novel strategies for achieving integration between scaffold and native cartilage.

\section{Designing Scaffolds to Promote CSI}

The characteristics of biomaterials are one of the foremost nonbiological factors that control CSI. A suitable scaffold would provide an optimal and proper architecture for cellular development and attachment. Scaffold properties is an important aspect in tissue regeneration, and resemblance of engineered cartilage to native tissue could enhance integration procedure. To achieve this kind of similarity, different strategies can be developed to manipulate biomaterial's surface chemistry, composition, morphology, and stiffness (Figure 2). For instance, it has been shown that by simulating tissue-like surface chemistry or stiffness, proliferation and growth of chondrocytes or differentiation of stem cells toward desired path can be improved [18-21]. The scaffold's biochemical composition and topographical features can facilitate desirable cellbiomaterial interactions and consequently promote CSI $[22,23]$. Below, we describe several features related to CSI that should be taken into consideration in designing and fabricating scaffolds.

2.1. Chemical Composition. The chemical composition of scaffolds can alter their physicochemical and mechanical properties and control particular cell/stem cell functions [24]. Researchers have yet to determine whether the ultrastructure or chemical composition of ECM plays a more significant role in stem cell phenotype/lineage determination. Nevertheless, it is crucial to consider the composition of native cartilage in order to establish the proper therapeutic approach. Proteomic analysis of cartilage showed that it is mostly composed of a mixture of highly glycosylated PGs and triple-helical collagens [25], and tensile integrity and remodeling of collagen network were precisely regulated 


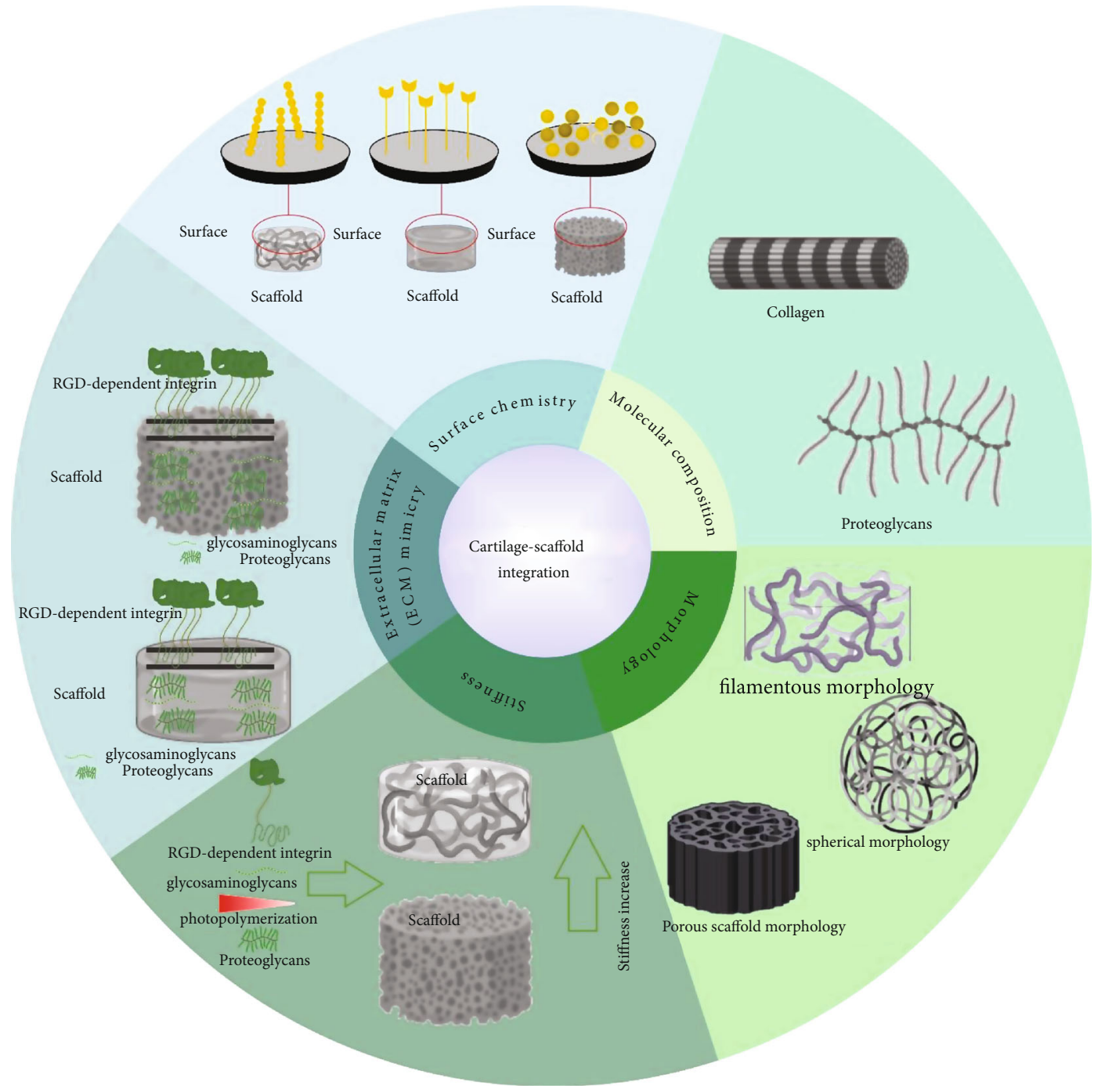

Figure 2: Cartilage-scaffold integration. Several parameters have been marked as significant agents in the CSI.

by collagen-interacting matrix molecules. Asanbaeva et al. reported that aggrecan has an inhibitory effect in adherence of collagen fibrils together and hence reduces tensile integrity of immature cartilage [26]. Collagenase delivery by means of nanofibrous scaffold has also been examined. The degenerative capacity of collagenase would result in greater tissue integration [27]. Applying chondroitinase $\mathrm{ABC}$ in an in vitro model of cartilage defect also improved integration of self-assembled articular cartilage to native cartilage [28]. Combination of trypsin treatment and delivery of heparin-binding insulin-like growth factor-1 (HBIGF-1) also stimulates cartilage integration and matrix biosynthesis [29]. Collagen crosslinking agents like lysyl oxidase also facilitated cartilage integration in vitro [30]. Thus, it can be suggested that designing scaffolds to eliminate potential extrafibrillar inhibitory molecules or addition of specific enzymes would reinforce collagen network construction and may result in more robust CSI. However, maintenance of enzymatic activity and controlled release of enzymes would be important under in vivo condition to drive desired results and avoid further tissue degeneration or prolonged inflammation at the same time.

Providing cells with ECM or ECM-derived components also seems promising in optimization of cellular microenvironment and maintenance of viable and proliferating cells which can result in tissue integration. However, it is important to consider the prominent role of different cell types in provoking this integration. It has been shown that combination of decellularized matrices with chondrocytes or other appropriate cell types would promote higher CSI in comparison to decellularized matrix alone [31, 32]. Barthold et al. demonstrated that recruiting ECM microparticles embedded in hyaluronic acid (HA) hydrogel improves chondrocyte migration through chemotaxis and so resulted in tissue integration [33]. It seems that strategies which promote collagen deposition by direct cell recruitment or indirect stimulation of cell migration would result in greater scaffold-cartilage integration.

Endless capacity of biomaterials for developing tissueadhesive scaffolds could be an attractive regenerative strategy 
to reach scaffold-cartilage integration [34-42]. In one of the early studies, multifunctional chondroitin sulfate was used as a bridge molecule to enhance attachment of poly (ethylene glycol) diacrylate (PEGDA) hydrogels to surrounding cartilage [17]. This adhesive hydrogel has also been examined in pilot clinical study and was promising when applied to the defect site in combination with microfracture surgery [43]. Photochemical crosslinking is a procedure that relies on rapid and durable bond formation at the scaffold-tissue interface and can cause more robust integration. Arvayo et al. reported the efficiency of phthalocyanine chloride tetrasulfonic acid (CASPc) and aluminum phthalocyanine chloride $(\mathrm{AlPc})$ on articular cartilage integration. Both photosensitizers could augment functional integration without changing cell viability [44]. Using exosome-encapsulated photoinduced imine crosslinking (PIC) hydrogels has led to in vitro and in vivo tissue integration as a result of reaction between aldehyde groups of the hydrogel and amino groups of cartilage [40]. Mohan et al. recruited microspheres to generate material and growth factor gradient which then were used to heal osteochondral defects in rabbit knees. The degree of tissue integration was well beyond the control group [45]. In a recent study, a positively charged elastinlike protein was electrostatically combined with chondroitin sulfate to produce a protein adhesive. This adhesive structure resulted in lateral tissue integration. Better chondrogenesis was also observed due to the release of chondroitin sulfate from electrostatic adhesive [46]. Traditional tissue adhesives may lack some vital aspects of constructive scaffold. These adhesive molecules are not specifically developed to reinforce cellular activities and also may not be suitable to fill large defects [47]. Hence, using adhesive scaffolds could greatly impact integration between scaffold and native cartilage and surpass the effects of traditional tissue glues.

2.2. Porosity. Many cellular behaviors such as attachment, cell-scaffold integration, and ECM secretion depend on the porosity and pore size of the scaffold [48]. An appropriate biomechanical and topological environment for cartilage regeneration could be provided via a well-designed porous scaffold. In a study by Matsiko et al., collagen-HA scaffolds seeded by MSCs were manufactured with pore sizes ranging from 94 to 300 microns. Matrix deposition, cell attachment, and chondrogenic gene expression increased in scaffolds with larger pore size [49]. The importance of scaffold porosity has been shown in an in vitro study of meniscus defect repair. In this study, preseeded porous scaffolds resulted in better tissue integration [50]. Porous chondrocyte-seeded honeycomb-like expandable gelatin scaffold is used to repair rabbit femoral condyle defect. Significant tissue regeneration and integration were observed in cell-seeded porous scaffolds in comparison to autologous chondrocyte implantation [51]. Ideal porosity can even lead to satisfactory integration of cell-free scaffolds with native tissue and development of convenient off-the-shelf products $[52,53]$. Pore size and density of scaffolds affect ECM integrity and maturity. Interconnected pores increase cell infiltration, ECM secretion, and collagen deposition which consequently could result in better CSI.
2.3. Mechanical Loading. Mechanotransduction pathways are fundamental for regulating matrix synthesis in loadbearing cartilage [54]. As a result, adjusted mechanical loading can impact scaffold-cartilage integration. It has been demonstrated that compressive loading augments GAGs and collagen synthesis in cell-seeded hydrogels $[55,56]$. However, constructs with poor mechanical properties in comparison to native cartilage can lead to abnormal stress accumulation on the latter part and failure of tissue integration $[57,58]$ due to altered mechanotransduction signaling. Mechanical stimulation could positively impact tissue integration through increasing collagen synthesis at scaffoldcartilage interface [59]. Yodmuang et al. hypothesized that mechanical discontinuity between scaffold and native cartilage could negatively impact integration. It has been shown that peripheral confinement of cartilage explants prior to compressive loading would increase scaffold-cartilage interface strength. Loading itself also augments GAG content in the scaffolds which follows by more scaffold-cartilage integration [60].

\section{Recruiting Cells to Achieve CSI}

Surrounding dead tissue in the defect edge which has lower cell density can impede integration of grafted tissue to native cartilage. To overcome this hurdle, supplying cells by means of scaffolds could positively affect integration. Since chondrocytes are viable part of the cartilage and responsible for ECM synthesis and henceforth tissue stiffness, their migration is a defining factor in cartilage integration. For example, Src-PLC $\gamma 1$-ERK1/2 signaling pathway affected chondrocyte migration in vitro and inhibition of this pathway resulted in lower interfacial integrative strength [61]. Nevertheless, migration of endogenous chondrocytes to defect site barely occurs in vivo [62]; therefore, cell delivery could be beneficial for superior cartilage integration. Here, we discuss strategies for delivering cellular component to achieve higher scaffold-cartilage integration.

3.1. Scaffold-Free Constructs. Cell therapy of cartilage defects is vastly investigated in preclinical and clinical settings [63]. Although the short-term outcomes of these studies are pretty compelling, only patients with minimal or surface cartilage injuries benefit from these therapies in long-term [64]. Park et al. have examined reparative capacity of chondrocyte spheroids and their self-produced ECM in a rabbit model. The constructs exhibited significant ECM accumulation in vitro and excellent integration with surrounding cartilage tissues in vivo [65]. Tuneability, injectability, and biomechanical superiority of human nasal chondrocytes (hNCs) have been confirmed by Gryadunova et al. Injecting hNC spheroids to the explants of bovine intervertebral disc has illustrated its great potential for regeneration of nucleus pulposus (NP) in a minimally invasive procedure [66]. Three dimensional (3D) scaffold-free hyaline cartilage constructs with clinically relevant size were derived from human embryonic stem cells (hESCs). These constructs were mechanically comparable with native cartilage and effectively integrate with human cartilage explants [67]. As 
mentioned earlier, cells are important components of integrative cartilage repair and their absence would halt tissue integration [31]. Although they are the main contributors to ECM synthesis and cartilage microenvironment maintenance, complete omission of scaffolds from regeneration process may result in poor CSI due to incompatible mechanical properties of native and newly formed cartilage.

3.2. Targeted Cell Delivery. Hypothetically, we can assume that targeted delivery of the chondrocytes or stem cells such as MSCs to the injury site would have positive impact on integration between newly formed tissue and native cartilage. The significance of targeted cell delivery (TCD) is accentuated by keeping in mind that tissue regeneration could be easily hampered as a result of cell senescence and death due to anoikis $[68,69]$. TCD is achieved by means of different surface functionalization procedures including antibody, peptide, selectin, and genetically mediated modifications which is reviewed elsewhere [70]. Li et al. exploited the targeting of membrane-modified MSCs to the injury site in which the transglutaminase- 2 overexpression is a hallmark and utilized as an anchor for modified MSCs. This targeted MSC delivery resulted in better histological scores and gene expression relevant to repair of in vivo cartilage defect [71]. Dual functionalization of PEG by transcyclooctene (TCO) and apoptotic binding peptide (ApoPep-1) led to the development of a crosslinker that binds to apoptotic chondrocytes of injured cartilage explant and methyltetrazine-bearing metabolically active chondrocytes through ApoPep-1 and TCO, respectively. This click chemistry-based pretargeting approach resulted in significant reduction of cartilage degeneration and increased ECM synthesis [72]. Since TCD is a developing approach for repairing cartilage defects, its integrative capacity is not well-studied yet, but is expected to be explored in early future. Theoretically, it can be inferred that precise delivering and attachment of cells to the defect area would have great regenerative impact and can boost ECM synthesis and tissue integration.

\section{The Role of Biomolecules in CSI}

Diverse biomolecules may facilitate integration of native cartilage with the implanted construct (Table 1). Under normal conditions, many regulatory molecules such as growth factors and cytokines act to maintain cartilage tissue homeostasis. These molecules mediate various functions in cell migration, attachment, proliferation, differentiation, and senescence [73]. It is presumed that the ability of cells to migrate towards the defect edge is partially responsible for CSI [74]. However, removal of damaged tissue around the edge of the lesion leaves behind necrotic tissue that acts like a barrier for cellular migration and impedes the desired integration with the host tissue. Chemotactic biomolecules can alleviate this negative condition by enhancing migration of endogenous chondrocytes. In an in vitro study, researchers assessed the chemotactic potential of platelet derived growth factor-bb (PDGF-bb), basic fibroblast growth factor (bFGF), or insulin-like growth factor 1 (IGF-1) combined with an enzymatic rinse to induce cell migration from bovine cartilage explants. It has been shown that brief collagenase treatment followed by supplementation of IGF-1 resulted in significantly increased chondrocyte population around the periphery of the explant [75]. Chemotactic effect of PDGF for chondrocytes was also confirmed in bovine meniscus and PDGF-coated scaffolds resulted in integrative repair of explants [76]. Interestingly, the results of another study confirmed that cartilage integration occurred by inducing chondrocyte migration towards the lesion's edge. Pabbruwe et al. sandwiched a porous bovine collagen scaffold seeded by nasal chondrocytes between two cartilage discs. After 40 days of culture with FGF-2, cell migration between two tissue surfaces across the collagenous membrane induced tissue integration and led to cartilaginous matrix deposition and disappearance of interface borders [77].

Growth factors may promote tissue integration by means of increasing chondrogenic differentiation. Smyth et al. hypothesized that platelet-rich plasma (PRP), as a cocktail of growth factors, might induce graft integration with the host tissue. To evaluate this, osteochondral grafts were treated with either PRP or saline solution before pressfitting into rabbit osteochondral lesions. The graft integration was significantly higher in the PRP-treated group [78]. It was assumed that osteochondral transplantation of MSCs is followed by PRP-induced chondrogenic differentiation. In a recent study, adipose-derived stem cells (ADSCs) were loaded in a thiolated gelatin/poly (ethylene glycol) diacrylate hydrogel with or without IGF-I. The composite hydrogels were then implanted into rabbit osteochondral defects. In contrast to the control group, neocartilage tissue was integrated with the adjacent native tissue in the presence of IGF-I. Incorporation of Coacervates (Coa) resulted in longterm release of IGF-I and even superior tissue integration [79]. Based on this study, the researchers presumed that sustained release of growth factors might provoke CSI. In this regard, Ren et al. fabricated a composite scaffold composed of porcine demineralized bone matrix and poly (alanine ethyl ester-co-glycine ethyl ester) phosphazene (PAGP) microspheres that contained TGF- $\beta 1$, IGF-1, and bone marrow-derived stem cells (BMSCs). Subcutaneous implantation of this construct in nude mice resulted in sustained release of GFs and chondrogenic differentiation of BMSCs with increased GAG deposition compared to the group without GFs. The neocartilage tissue was integrated with the host tissue and exhibited remarkable biomechanical properties that approximated native tissue [80].

Cell recruitment through chemotaxis is another approach to augment tissue integration. Luo et al. used the chemotactic effects of TGF- $\beta 3$ and mechanogrowth factor (MGF) to recruit stem cells at the injury site. Silk fibroin scaffolds were functionalized with either TGF- $\beta 3$ (ST group) or TGF- $\beta 3$ / MGF (STM group) and were then implanted into rabbit osteochondral defects. The results showed that STM scaffolds demonstrated superior integration with the host tissue. MGF and TGF- $\beta 3$ synergistically increased endogenous stem cell recruitment. Moreover, MGF downregulated collagen type I expression, which resulted in less fibrocartilage formation and better tissue repair [81]. 
TABLE 1: Biomolecules in cartilage integration.

\begin{tabular}{|c|c|c|c|}
\hline No. & $\begin{array}{c}\text { Recruited } \\
\text { biomolecule }\end{array}$ & Result & Reference \\
\hline 1 & FGF-2 & $\begin{array}{l}\text { Increased GAG and type II collagen biosynthesis. Proliferation and differentiation of } \\
\text { chondrocytes }\end{array}$ & $\begin{array}{c}{[77,} \\
114-117]\end{array}$ \\
\hline 2 & IGF-1 & $\begin{array}{c}\text { Stimulation of proteoglycan synthesis, chondrocyte proliferation, and cell homing. Improved } \\
\text { histologic appearance in rabbit full-thickness cartilage defect }\end{array}$ & $\begin{array}{c}{[75,79,} \\
118-123]\end{array}$ \\
\hline 3 & FGF-18 & Increased hyaline-cartilage production & {$[124,125]$} \\
\hline 4 & Kartogenin & Intact cartilage regeneration & {$[126]$} \\
\hline 5 & TGF- $\beta 1$ & Improved chondrogenic regeneration and cartilage integrity in a rabbit model & [127] \\
\hline 6 & TGF- $\beta 1 /$ IGF-1 & Chondrogenic differentiation, GAG deposition, and neocartilage integration to host tissue & {$[80]$} \\
\hline 7 & TGF- $\beta 3$ & $\begin{array}{l}\text { Extracellular matrix formation by fibrochondrocytes of meniscus. Endogenous stem cell } \\
\text { recruitment and in situ cartilage regeneration }\end{array}$ & {$[81,128]$} \\
\hline 8 & TGF- $\beta 3 /$ kartogenin & $\begin{array}{l}\text { Promotion of chondrogenesis and cartilage regeneration by synergistic effect and improved } \\
\text { integrity in rabbit models }\end{array}$ & [129] \\
\hline 9 & PRP & Enhanced chondrocyte proliferation and redifferentiation. Increase matrix accumulation & $\begin{array}{l}{[78,130,} \\
131]\end{array}$ \\
\hline 10 & BMHP & Stem cell recruitment to the defect site and neocartilage similarity to native tissue & {$[82]$} \\
\hline 11 & $\begin{array}{l}\text { KLPP self- } \\
\text { assembling peptide }\end{array}$ & Recruitment of endogenous chondrocytes and promotion of tissue integration & [83] \\
\hline
\end{tabular}

BMHP: bone marrow homing peptide.

Recently, peptide biomolecules have been used to recruit cells and promote tissue integration. Bone marrow homing peptide (BMHP) has chemotactic effects on BMSCs homing. $\mathrm{Lu}$ et al. utilized BMHP-functionalized acellular matrixderived scaffolds to enhance cartilage regeneration. The scaffolds were implanted into rabbit full-thickness cartilage defects. Six months postsurgery, defects were filled up with neocartilage tissue that had a smooth surface similar to the native tissue [82]. Lv et al. attempted to integrate the neocartilage with the host tissue by using two custom-designed functionalized self-assembling peptide hydrogels, KLD-12 and KLPP [83]. These peptides possess a functionalized nanostructure with low cytotoxicity that induces chondrocyte and BMSC migration. KLPP has better performance against KLD-12 in vitro. Although, osteochondral defects in the KLD-12 and KLPP groups were suitably filled with neocartilage tissue compared to the control group, and the KLPP group exhibited the most desirable integration with surrounding host tissue. It is concluded that the KLPP peptide hydrogel significantly recruit endogenous chondrocytes and BMSCs and promote tissue integration and cartilage repair.

In brief, growth factors might direct tissue integration by inducing chondrocyte migration towards lesion peripheries, increasing chondrogenic differentiation of stem cells, or recruitment of endogenous stem cells to the area of the lesion. In this context, it is of utmost importance to understand the mechanisms of biomolecule-induced cartilage integration, since any successful regenerative scaffold-based strategy for cartilage repair is contingent upon suitable integration of the construct and the host tissue.

\section{Novel Strategies for Improvement of CSI}

5.1. Bioprinting. $3 \mathrm{D}$-printing is an intricate method that could improve scaffold integration with surrounding tissue because of the anticipated anisotropic properties of the printed tissue and the possibility of personalized designation of the constructs. In one of the early studies, a poly (ethylene glycol) dimethacrylate (PEGDMA) bioink that contained human chondrocytes firmly attached to the surrounding osteochondral plug after printing. It was hypothesized that direct printing of the hydrogels to the defect site would cause more ECM production and better tissue integration [84]. Shim et al. developed a heterogeneous scaffold by multilayer printing of atelocollagen and supramolecular HA, which was seeded with human turbinate-derived MSCs. They observed cartilaginous tissue formation and integration with adjacent tissues and lack of noticeable inflammation two months postimplantation into rabbit knee joint defects [85]. A layered osteochondral scaffold that consisted of a cartilage layer of gelatin/alginate and a bone layer of gelatin/alginate/ hydroxyapatite was seeded with bone marrow stem cells and $3 \mathrm{D}$-bioprinted. It was assumed that effective integration of the printed constructs with native rabbit cartilage was due to the precise biomimetic structure of the scaffolds [86]. In an in vitro study by Daly and Kelly, stratified cartilage structure was achieved due to inkjet bioprinting of cellular spheroids that contained a defined number of MSCs and chondrocytes into preprinted PCL microchambers. This strategy resulted in condensation and organization of cellular spheroids in the PCL microchambers and the formation of an osteochondral construct. This approach facilitated the integration of bone and cartilage regions in these constructs [87]. Gong et al. investigated the effect of bilayered printed scaffolds in repairing an osteochondral injury in a rabbit model. The upper and lower layers were composed of interleukin 4- (IL4-) loaded GelMa and PCL/HA, respectively. IL-4 was used because of its anti-inflammatory properties. There was significantly more tissue integration in the IL4-loaded constructs 16 weeks after implantation compared 
to the control groups [88]. Recently, Sun et al. reported that 3D-bioprined anisotropic dual-factor (transforming growth factor beta-3 [TGF- $\beta 3$ ] and bone morphogenetic protein 4 [BMP4] in PLGA microspheres) releasing constructs comprised of PCL and MSC-laden hydrogels led to better cartilage regeneration. These constructs were grafted in knee cartilage defects of a rabbit model. At the six-month follow-up, the researchers noted the similarity of these constructs to normal cartilage in their appearance and gradient structure as well as better microvessel formation that resulted in more robust integration of the graft with surrounding tissue compared to the control group [89]. The regenerative capacity of a functionalized tyramine/methacryloyl gelatin (GelMa-Tyr) bioink was investigated for ex vivo cartilage repair. Unlike most ex vivo studies, the adhesion of printed constructs to surrounding native tissue was evaluated. The presence of tyramine in this hydrogel augmented tissue adhesion by 15-fold compared to GelMa alone [90]. Bioprinting is also utilized to produce an anisotropic construct that benefits from different pore sizes and mimics the gradient structure of native cartilage. Fourlayer BMSC-laden bioprinted construct with various pore sizes in each layer has led to better tissue integration and repair in rabbit knee defect [91]. In another study, in situ bioprinting of MSC-laden hyaluronic acid methacrylate(HAMA-) GelMa bioink via Biopen increased tissue integration in a sheep model of knee cartilage defect [92]. However, there was a brief 8 -week follow-up that might prevent conclusions about the efficacy of this procedure. Roboticassisted in situ 3D-bioprinting of HAMA bioink that was reinforced with an acrylate-terminated 4-armed PEG crosslinker in a rabbit model of cartilage defect was promising in terms of hyaline-like cartilage formation and tissue integration after 12 weeks of follow-up [93]. Most of the abovementioned studies utilized photocrosslinking of the bioinks as the principal procedure for making durable constructs at the defect site. As a result, it can be inferred that this process could be of importance in the future of cartilage bioprinting. In general, the precision of the bioprinting method and, in particular, in situ bioprinting could revolutionize CTE. Additional integrative studies would lead to more chemically designed bioinks and better integration of the implant with native cartilage. The emergence of $4 \mathrm{D}$-printing could be another field of development for CTE. In this approach, 3D-printed constructs would change in accordance with stimulation from the nearby environment. In conclusion, better CSI could be attained with the design of new biomaterials that respond to injured cartilage environment and act suitably to repair the defect.

5.2. Extracellular Vesicles. A recent biomolecule-based strategy that included the use of EVs was explored to stimulate cartilage-cartilage integration. EVs are bilayer lipid particles naturally secreted by various types of cells. These secreted membrane-enclosed vesicles range from 10 to $20 \mathrm{~nm}$ up to $10 \mu$ in diameter and are categorized into exosomes (50$200 \mathrm{~nm}$ ), microvesicles (MVs) (30-150 nm), and apoptotic bodies (500-1000 nm). EVs mediate intercellular communication and maintain physiological homeostasis through delivery of biological molecules (RNA, DNA, proteins, lipids, metabolites, and some organelles) [94].

EVs have many different adhesion molecules that help them to interact with ECM components and cells. In addition, they can induce the production of fibronectin, collagen type II (COL2A1), and ECM components as well as promote expression of SRY-related HMG-box-9 (Sox-9), proteoglycan 4 (PRG4), aggrecan (ACAN), and cartilage oligomeric matrix protein (or thrombospondin 5) genes [95-100]. EVs can also improve ECM synthesis by reducing the expression of type X collagen (COL10A1) matrix metalloproteinases-1/ $3 / 13$, runt-related transcription factor 2, aggrecanase-5, and wingless-type MMTV integration site family, member $5 \mathrm{~A}$ genes [96, 101-104]. These features may lead to improvements in cartilage tissue integration. Moreover, EVs participate in cartilage repair, regeneration, and mineralization processes that eventually promote the integration of cartilage tissue [105].

Another characteristic of EVs that can be attributed to the induction of cartilage integrity is the polarization from M1 to M2 in immune cells, including monocytes and macrophages, as well as promotion of the immune modulation effects by in vitro and in vivo secretion of cytotoxic agents [106]. EVs may be of benefit in the integration of cartilage tissue because of their ability to regulate the AKT and ERK signaling pathways and their capacity to promote the proliferation and infiltration of cartilage tissues [107].

Recent findings suggest that EVs obtained from stem cells may be a promising strategy that could not only ameliorate cartilage healing but also stimulate cartilage integration [40, 108-112]. The results of an animal study on rats with trochlear grooves of both distal femurs showed that exosomes derived from human embryonic MSCs might improve complete cartilage regeneration with good surface arrangement, complete connection to adjacent cartilage, and ECM deposition 12 weeks after surgery, thus ameliorating the probability of cartilage integrity and reducing fibrous tissue formation [107]. In another study, EVs released by BMSCs decreased proinflammatory gene expressions, revoked the TNF-alpha-dependent upregulation of proinflammatory interleukins and COX2, and suppressed TNFalpha-mediated collagenase activity. They also improved cartilage integration by the promotion of COL II and PG synthesis in osteoarthritis (OA) chondrocytes. Treatment with BMSC EVs led to the suppression of hypertrophic factors gene expressions, such as runt-related transcription factor 2 (RUNX2), type X collagen, and alkaline phosphatase (ALP), which led to an improvement in the cartilage integrity process .

There is evidence that exosomes from BMSCs through kartogenin (KGN) preconditioning (KGN-BMSC-Exosomes) suppress fibrous tissue formation and stimulate the metabolic activity of chondrocytes via promotion of cell proliferation and migration and cartilage matrix production. Thereby, they reduce COL I and c-Myc expressions and, therefore, amplify the probability of chondrocyte maturation and increase COL II, s-GAG, lubricin, and Sox-9 synthesis [99]. With this evidence, KGN-BMSC-exosomes can be impregnated to tissue-engineered scaffolds to improve 
cartilage repair and integrity. Human umbilical cord-derived mesenchymal stem cell-derived small EVs (hUC-MSCssEVs) considerably induced migration, proliferation, and differentiation of chondrocytes. Therefore, they ameliorated the rate of cartilage integrity and increased cartilage tissue regeneration [109]. Additionally, intra-articular administration of EVs secreted by chondrogenic progenitor cells from $\mathrm{MRL} / \mathrm{MpJ}$ superhealer mice demonstrated a beneficial effect on the proliferation and migration of murine chondrocytes, which might lead to amelioration in the cartilage regeneration [113].

All of the mentioned data show that EVs could be used as suitable candidates for cartilage tissue integrity because of the synthesis of extracellular matrix components, such as fibronectin and collagen type II, as well as their ability to ameliorate the cartilage degradation through inhibition of inflammatory mechanism.

\section{Concluding Remarks and Future Trends}

Despite notable achievements in utilizing novel biomaterials/techniques in CTE, the long-term integration of regenerated hyaline tissues with native cartilage remains elusive and needs urgent consideration. Development of new biomaterials/adhesives to precisely mimic natural cartilage structure and mechanical properties is an undeniable demand for achieving higher order biomechanical integration. On the other hand, apoptosis and necrosis of already rare chondrocytes at the edge of defect is a recurring issue which is partly responsible for lack of proper integration. This hurdle may be overcome by targeted cell delivery to somehow bridging the gap by cell migration and consequently ECM synthesis and metabolism. The precise control of physical or biochemical cues in novel engineered scaffolds could regulate ECM deposition and lead to CSI. The ability to build a native-like gradient of relevant biomolecules can also be beneficial in encouraging cell migration towards the defect edges and result in more robust integration. Stateof-the-art in situ 3D-bioprinting is also a promising upcoming trend since it can intricately mimic different aspects of ideal construct. Also, long-term assessments of preclinical and clinical trials could be more informative and ultimately beneficial for resolving integration challenge.

\section{Abbreviations}

$\begin{array}{ll}\text { ECM: } & \text { Extracellular matrix } \\ \text { GAGs: } & \text { Glycosaminoglycans } \\ \text { PGs: } & \text { Proteoglycans } \\ \text { CTE: } & \text { Cartilage tissue engineering } \\ \text { MSCs: } & \text { Mesenchymal stem cells } \\ \text { GFs: } & \text { Growth factors } \\ \text { PLGA: } & \text { Polyethylene glycol, poly (lactide-co- } \\ & \text { glycolic) acid } \\ \text { PCL: } & \text { Polycaprolactone } \\ \text { 3D: } & \text { Three-dimensional } \\ \text { EVs: } & \text { Extracellular vesicles } \\ \text { HA: } & \text { Hyaluronic acid } \\ \text { HAS: } & \text { Human serum albumin }\end{array}$

PLL:

CASPc:

AlPc:

PEGDMA:

IL4:

TGF- $\beta 3$ :

BMP4:

GelMa-Tyr:

HAMA:

PDGF-bb:

bFGF:

IGF-1:

FGF2:

PRP:

ADSCs:

COL II:

PAGP:

BMSCs:

MGF:

BMHP:

LPP:

ICRS:

MVs:

OA:

RUNX2:

ALP:

KGN:

hUC-MSCs-sEVs: Human umbilical cord-derived mesenchymal stem cell-derived small EVs.

\section{Data Availability}

Data sharing is not applicable to this article as no datasets were generated or analyzed during the current study.

\section{Conflicts of Interest}

The authors have no competing interests.

\section{Authors' Contributions}

SJ, AE, MS, and SH designed the concept. SJ, AE, MS, SHJ, and FZ searched the literature. SJ, AE, MS, SHJ, FZ, and MA wrote the manuscript. MS created the figures. $\mathrm{SH}$ revised the manuscript. The authors read and approved the final manuscript. Sahar Jelodari, Amin Ebrahimi Sadrabadi and Fatemeh Zarei contributed equally to this work.

\section{References}

[1] Y. Luo, D. Sinkeviciute, Y. He et al., "The minor collagens in articular cartilage," Protein \& Cell, vol. 8, no. 8, pp. 560-572, 2017.

[2] A. J. Sutherland, G. L. Converse, R. A. Hopkins, and M. S. Detamore, "The bioactivity of cartilage extracellular matrix in articular cartilage regeneration," Advanced Healthcare Materials, vol. 4, no. 1, pp. 29-39, 2015. 
[3] S. Tew, S. Redman, A. Kwan et al., "Differences in repair responses between immature and mature cartilage," Clinical Orthopaedics and Related Research, vol. 391, pp. S142-S152, 2001.

[4] R. Dorotka, U. Bindreiter, K. Macfelda, U. Windberger, and S. Nehrer, "Marrow stimulation and chondrocyte transplantation using a collagen matrix for cartilage repair," Osteoarthritis and Cartilage, vol. 13, no. 8, pp. 655-664, 2005.

[5] E. Solheim, J. Hegna, J. Øyen, T. Harlem, and T. Strand, "Results at 10 to 14 years after osteochondral autografting (mosaicplasty) in articular cartilage defects in the knee," The Knee, vol. 20, no. 4, pp. 287-290, 2013.

[6] B. McCoy and A. Miniaci, "Osteochondral autograft transplantation/mosaicplasty," The Journal of Knee Surgery, vol. 25, no. 2, pp. 99-108, 2012.

[7] J. A. Buckwalter and J. A. Martin, "Osteoarthritis," Advanced Drug Delivery Reviews, vol. 58, no. 2, pp. 150-167, 2006.

[8] A. Bedi, B. T. Feeley, and R. J. Williams 3rd, "Management of articular cartilage defects of the knee," The Journal of Bone and Joint Surgery. American Volume, vol. 92, no. 4, pp. 994-1009, 2010.

[9] E. C. Beck, M. Barragan, M. H. Tadros, S. H. Gehrke, and M. S. Detamore, "Approaching the compressive modulus of articular cartilage with a decellularized cartilage-based hydrogel," Acta Biomaterialia, vol. 38, pp. 94-105, 2016.

[10] S. L. Francis, C. Di Bella, G. G. Wallace, and P. F. M. Choong, "Cartilage tissue engineering using stem cells and bioprinting technology-barriers to clinical translation," Frontiers in Surgery, vol. 5, p. 70, 2018.

[11] M. K. Mamidi, A. K. Das, Z. Zakaria, and R. Bhonde, "Mesenchymal stromal cells for cartilage repair in osteoarthritis," Osteoarthritis and Cartilage, vol. 24, no. 8, pp. 1307-1316, 2016.

[12] S. P. Nukavarapu and D. L. Dorcemus, "Osteochondral tissue engineering: current strategies and challenges," Biotechnology Advances, vol. 31, no. 5, pp. 706-721, 2013.

[13] B. Balakrishnan and R. Banerjee, "Biopolymer-based hydrogels for cartilage tissue engineering," Chemical Reviews, vol. 111, no. 8, pp. 4453-4474, 2011.

[14] S. L. Vega, M. Y. Kwon, and J. A. Burdick, "Recent advances in hydrogels for cartilage tissue engineering," European Cells \& Materials, vol. 33, pp. 59-75, 2017.

[15] J. Yang, Y. S. Zhang, K. Yue, and A. Khademhosseini, "Cellladen hydrogels for osteochondral and cartilage tissue engineering," Acta Biomaterialia, vol. 57, pp. 1-25, 2017.

[16] F. Shapiro, S. Koide, and M. J. Glimcher, "Cell origin and differentiation in the repair of full-thickness defects of articular cartilage," The Journal of Bone \& Joint Surgery, vol. 75, no. 4, pp. 532-553, 1993.

[17] D.-A. Wang, S. Varghese, B. Sharma et al., "Multifunctional chondroitin sulphate for cartilage tissue-biomaterial integration," Nature Materials, vol. 6, no. 5, pp. 385-392, 2007.

[18] X. Punet, R. Mauchauffé, M. I. Giannotti et al., "Enhanced cell-material interactions through the biofunctionalization of polymeric surfaces with engineered peptides," Biomacromolecules, vol. 14, no. 8, pp. 2690-2702, 2013.

[19] T.-T. Yu, F.-Z. Cui, Q.-Y. Meng et al., "Influence of surface chemistry on adhesion and Osteo/odontogenic differentiation of dental pulp stem cells," ACS Biomaterials Science \& Engineering, vol. 3, no. 6, pp. 1119-1128, 2017.
[20] N. L. Bergholt, M. Foss, A. Saeed et al., "Surface chemistry, substrate, and topography guide the behavior of human articular chondrocytes cultured In Vitro," Journal of Biomedical Materials Research. Part A, vol. 106, no. 11, pp. 2805-2816, 2018.

[21] H. Lv, H. Wang, Z. Zhang et al., "Biomaterial stiffness determines stem cell fate," Life Sciences, vol. 178, pp. 42-48, 2017.

[22] M. A. Bilton, A. Thambyah, and R. J. Clarke, "How changes in interconnectivity affect the bulk properties of articular cartilage: a fibre network study," Biomechanics and Modeling in Mechanobiology, vol. 17, no. 5, pp. 1297-1315, 2018.

[23] Y. Qi, W. Zhang, G. Li et al., “An oriented-collagen scaffold including Wnt5a promotes osteochondral regeneration and cartilage interface integration in a rabbit model," The FASEB Journal, vol. 34, no. 8, pp. 11115-11132, 2020.

[24] A. Martinez, M. D. Blanco, N. Davidenko, and R. E. Cameron, "Tailoring chitosan/collagen scaffolds for tissue engineering: effect of composition and different crosslinking agents on scaffold properties," Carbohydrate Polymers, vol. 132, pp. 606-619, 2015.

[25] J. Piltti, J. Häyrinen, H. M. Karjalainen, and M. J. Lammi, "Proteomics of chondrocytes with special reference to phosphorylation changes of proteins in stretched human chondrosarcoma cells," Biorheology, vol. 45, no. 3-4, pp. 323-335, 2008.

[26] A. Asanbaeva, J. Tam, B. L. Schumacher, S. M. Klisch, K. Masuda, and R. L. Sah, "Articular cartilage tensile integrity: modulation by matrix depletion is maturation-dependent," Archives of Biochemistry and Biophysics, vol. 474, no. 1, pp. 175-182, 2008.

[27] F. Qu, J.-M. G. Lin, J. L. Esterhai, M. B. Fisher, and R. L. Mauck, "Biomaterial-mediated delivery of degradative enzymes to improve meniscus integration and repair," Acta Biomaterialia, vol. 9, no. 5, pp. 6393-6402, 2013.

[28] J. M. Link, J. C. Hu, and K. A. Athanasiou, "Chondroitinase $\mathrm{ABC}$ enhances integration of self-assembled articular cartilage, but its dosage needs to be moderated based on neocartilage maturity," Cartilage, vol. 13, no. 2, pp. 672S-683S, 2021.

[29] P. H. Liebesny, K. Mroszczyk, H. Zlotnick et al., "Enzyme pretreatment plus locally delivered HB-IGF-1 stimulate integrative cartilage RepairIn Vitro," Tissue Engineering Part A, vol. 25, no. 17-18, pp. 1191-1201, 2019.

[30] A. A. Athens, E. A. Makris, and J. C. Hu, "Induced collagen cross-links enhance cartilage integration," PLoS One, vol. 8, no. 4, article e60719, 2013.

[31] Y. Zhang, G. Feng, G. Xu, and Y. Qi, "Microporous acellular extracellular matrix combined with adipose-derived stem cell sheets as a promising tissue patch promoting articular cartilage regeneration and interface integration," Cytotherapy, vol. 21, no. 8, pp. 856-869, 2019.

[32] G. M. Peretti, T. J. Gill, J. W. Xu, M. A. Randolph, K. R. Morse, and D. J. Zaleske, "Cell-based therapy for meniscal repair: a large animal study," The American Journal of Sports Medicine, vol. 32, no. 1, pp. 146-158, 2004.

[33] J. E. Barthold, B. M. St Martin, S. L. Sridhar et al., "Recellularization and integration of dense extracellular matrix by percolation of tissue microparticles," Advanced Functional Materials, vol. 31, no. 35, article 2103355, 2021.

[34] C. Scotti, D. Wirz, F. Wolf et al., "Engineering human cellbased, functionally integrated osteochondral grafts by biological bonding of engineered cartilage tissues to bony scaffolds," Biomaterials, vol. 31, no. 8, pp. 2252-2259, 2010. 
[35] K. W. Ng, F. Wanivenhaus, T. Chen et al., "A novel macroporous polyvinyl alcohol scaffold promotes chondrocyte migration and Interface formation in an In Vitro cartilage defect model," Tissue Engineering Part A, vol. 18, no. 11-12, pp. 1273-1281, 2012.

[36] L. S. Moreira Teixeira, S. Bijl, V. V. Pully et al., "Self-attaching and cell-attracting in-situ forming dextran-tyramine conjugates hydrogels for arthroscopic cartilage repair," Biomaterials, vol. 33, no. 11, pp. 3164-3174, 2012.

[37] C. Wiltsey, P. Kubinski, T. Christiani et al., "Characterization of injectable hydrogels based on poly( $\mathrm{N}$-isopropylacrylamide)-g-chondroitin sulfate with adhesive properties for nucleus pulposus tissue engineering," Journal of Materials Science: Materials in Medicine, vol. 24, no. 4, pp. 837-847, 2013.

[38] B. Balakrishnan, N. Joshi, A. Jayakrishnan, and R. Banerjee, "Self-crosslinked oxidized alginate/gelatin hydrogel as injectable, adhesive biomimetic scaffolds for cartilage regeneration," Acta Biomaterialia, vol. 10, no. 8, pp. 3650-3663, 2014.

[39] X. Liu, Y. Yang, X. Niu et al., "An in situ photocrosslinkable platelet rich plasma - complexed hydrogel glue with growth factor controlled release ability to promote cartilage defect repair," Acta Biomaterialia, vol. 62, pp. 179-187, 2017.

[40] X. Liu, Y. Yang, Y. Li et al., "Integration of stem cell-derived exosomes with in situ hydrogel glue as a promising tissue patch for articular cartilage regeneration," Nanoscale, vol. 9, no. 13, pp. 4430-4438, 2017.

[41] L. Han, M. Wang, P. Li et al., "Mussel-inspired tissueadhesive hydrogel based on the polydopamine-chondroitin sulfate complex for growth-factor-free cartilage regeneration," ACS Applied Materials \& Interfaces, vol. 10, no. 33, pp. 28015-28026, 2018.

[42] O. Berberich, J. Blöhbaum, S. Hölscher-Doht et al., "Catechol-modified poly(oxazoline)s with tunable degradability facilitate cell invasion and lateral cartilage integration," Journal of Industrial and Engineering Chemistry, vol. 80, pp. 757769, 2019.

[43] B. Sharma, S. Fermanian, M. Gibson et al., "Human cartilage repair with a photoreactive adhesive-hydrogel composite," Science Translational Medicine, vol. 5, no. 167, p. 167ra6, 2013.

[44] A. L. Arvayo, I. J. Wong, J. L. Dragoo, and M. E. Levenston, "Enhancing integration of articular cartilage grafts via photochemical bonding," Journal of Orthopaedic Research, vol. 36, no. 9, pp. 2406-2415, 2018.

[45] N. Mohan, N. H. Dormer, K. L. Caldwell, V. H. Key, C. J. Berkland, and M. S. Detamore, "Continuous gradients of material composition and growth factors for effective regeneration of the osteochondral interface," Tissue Engineering Part A, vol. 17, no. 21-22, pp. 2845-2855, 2011.

[46] J. Zhang, B. Li, J. Zuo et al., "An engineered protein adhesive with properties of tissue integration and controlled release for efficient cartilage repair," Advanced Healthcare Materials, vol. 10, no. 12, p. 2100109, 2021.

[47] S. Chen, C. J. Gil, L. Ning et al., "Adhesive tissue engineered scaffolds: mechanisms and applications," Frontiers in Bioengineering and Biotechnology, vol. 9, p. 683079, 2021.

[48] O. Oliviero, M. Ventre, and P. A. Netti, "Functional porous hydrogels to study angiogenesis under the effect of controlled release of vascular endothelial growth factor," Acta Biomaterialia, vol. 8, no. 9, pp. 3294-3301, 2012.
[49] A. Matsiko, J. P. Gleeson, and F. J. O'Brien, "Scaffold mean pore size influences mesenchymal stem cell chondrogenic differentiation and matrix deposition," Tissue Engineering Part A, vol. 21, no. 3-4, pp. 486-497, 2015.

[50] L. C. Ionescu and R. L. Mauck, "Porosity and cell preseeding influence electrospun scaffold maturation and meniscus integration in vitro," Tissue Engineering Part A, vol. 19, no. 3-4, pp. 538-547, 2013.

[51] C.-C. Wang, K.-C. Yang, K.-H. Lin et al., "Expandable Scaffold Improves Integration of Tissue-Engineered Cartilage: An In Vivo Study in a Rabbit Model," Tissue Engineering Part A, vol. 22, no. 11-12, pp. 873-884, 2016.

[52] Y. Dai, G. Liu, L. Ma, D. Wang, and C. Gao, "Cell-free macroporous fibrin scaffolds for in situ inductive regeneration of full-thickness cartilage defects," Journal of Materials Chemistry B, vol. 4, no. 25, pp. 4410-4419, 2016.

[53] X. Wu, J. Ding, P. Xu et al., "A cell-free ROS-responsive hydrogel/oriented poly(lactide-co-glycolide) hybrid scaffold for reducing inflammation and restoring full-thickness cartilage defects In Vivo," Biomedical Materials, vol. 16, no. 6, p. 064101, 2021.

[54] J. D. Szafranski, A. J. Grodzinsky, E. Burger, V. Gaschen, H.-H. Hung, and E. B. Hunziker, "Chondrocyte mechanotransduction: effects of compression on deformation of intracellular organelles and relevance to cellular biosynthesis," Osteoarthritis and Cartilage, vol. 12, no. 12, pp. 937946, 2004.

[55] R. L. Mauck, M. A. Soltz, C. C. B. Wang et al., "Functional tissue engineering of articular cartilage through dynamic loading of chondrocyte-seeded agarose gels," Journal of Biomechanical Engineering, vol. 122, no. 3, pp. 252-260, 2000.

[56] P. Wu, E. DeLassus, D. Patra, W. Liao, and L. J. Sandell, "Effects of serum and compressive loading on the cartilage matrix synthesis and spatiotemporal deposition around chondrocytes in 3D culture," Tissue Engineering Part A, vol. 19, no. 9-10, pp. 1199-1208, 2013.

[57] A. Vahdati and D. R. Wagner, "Finite element study of a tissue-engineered cartilage transplant in human tibiofemoral joint," Computer Methods in Biomechanics and Biomedical Engineering, vol. 15, no. 11, pp. 1211-1221, 2012.

[58] T. Chen, M. M. McCarthy, H. Guo, R. Warren, and S. A. Maher, "The scaffold-articular cartilage interface: a combined In Vitro and in silico analysis under controlled loading conditions," Journal of Biomechanical Engineering, vol. 140, no. 9, pp. 0910021-0910027, 2018.

[59] J. S. Theodoropoulos, A. J. N. DeCroos, M. Petrera, S. Park, and R. A. Kandel, "Mechanical stimulation enhances integration in an in vitro model of cartilage repair," Knee Surgery, Sports Traumatology, Arthroscopy, vol. 24, no. 6, pp. 20552064, 2016.

[60] S. Yodmuang, H. Guo, C. Brial et al., "Effect of interface mechanical discontinuities on scaffold-cartilage integration," Journal of Orthopaedic Research, vol. 37, no. 4, pp. 845-854, 2019.

[61] Y. Lu, Y. Xu, Z. Yin, X. Yang, Y. Jiang, and J. Gui, "Chondrocyte migration affects tissue-engineered cartilage integration by activating the signal transduction pathways involving Src, PLC $\gamma 1$, and ERK1/2," Tissue Engineering Part A, vol. 19, no. 21-22, pp. 2506-2516, 2013.

[62] M. K. Lotz, S. Otsuki, S. P. Grogan, R. Sah, R. Terkeltaub, and D. D'Lima, "Cartilage cell clusters," Arthritis and Rheumatism, vol. 62, no. 8, pp. 2206-2218, 2010. 
[63] C. H. Hulme, J. Perry, H. S. McCarthy et al., "Cell therapy for cartilage repair," Emerging Topics in Life Sciences, vol. 5, no. 4, pp. 575-589, 2021.

[64] X. Nie, Y. J. Chuah, W. Zhu, P. He, Y. Peck, and D.-A. Wang, "Decellularized tissue engineered hyaline cartilage graft for articular cartilage repair,” Biomaterials, vol. 235, p. 119821, 2020.

[65] I. S. Park, R. L. Jin, H. J. Oh et al., "Sizable scaffold-free tissueengineered articular cartilage construct for cartilage defect repair," Artificial Organs, vol. 43, no. 3, pp. 278-287, 2019.

[66] A. Gryadunova, J. Kasamkattil, M. H. P. Gay et al., "Nose to spine: spheroids generated by human nasal chondrocytes for scaffold-free nucleus pulposus augmentation," Acta Biomaterialia, vol. 134, pp. 240-251, 2021.

[67] L. A. Griffith, K. M. Arnold, B. G. Sengers, R. S. Tare, and F. D. Houghton, "A scaffold-free approach to cartilage tissue generation using human embryonic stem cells," Scientific Reports, vol. 11, no. 1, p. 18921, 2021.

[68] J. Li and M. Pei, "Cell Senescence: A challenge in cartilage engineering and regeneration," Tissue Engineering Part B: Reviews, vol. 18, no. 4, pp. 270-287, 2012.

[69] D. J. Mooney and H. Vandenburgh, "Cell delivery mechanisms for tissue repair," Cell Stem Cell, vol. 2, no. 3, pp. 205-213, 2008.

[70] N. Nasiri, S. Hosseini, M. Alini, A. Khademhosseini, and M. B. Eslaminejad, "Targeted cell delivery for articular cartilage regeneration and osteoarthritis treatment," Drug Discovery Today, vol. 24, no. 11, pp. 2212-2224, 2019.

[71] H. Li, Y. Jin, Y. Zhao et al., "Targeted cell therapy for partialthickness cartilage defects using membrane modified mesenchymal stem cells by transglutaminase 2," Biomaterials, vol. 275, p. 120994, 2021.

[72] C. M. Co, S. Izuagbe, J. Zhou et al., "Click chemistry-based pre-targeting cell delivery for cartilage regeneration," Regenerative Biomaterials, vol. 8, no. 3, 2021.

[73] M. M. Pillai, J. Gopinathan, R. Selvakumar, and A. Bhattacharyya, "Human knee meniscus regeneration strategies: a review on recent advances," Current Osteoporosis Reports, vol. 16, no. 3, pp. 224-235, 2018.

[74] I. M. Khan, S. J. Gilbert, S. K. Singhrao, V. C. Duance, and C. W. Archer, "Cartilage integration: evaluation of the reasons for failure of integration during cartilage repair. A review," European Cells \& Materials, vol. 16, pp. 26-39, 2008.

[75] A. J. McGregor, B. G. Amsden, and S. D. Waldman, "Chondrocyte repopulation of the zone of death induced by osteochondral harvest," Osteoarthritis and Cartilage, vol. 19, no. 2, pp. 242-248, 2011.

[76] K. I. Lee, M. Olmer, J. Baek, D. D. D'Lima, and M. K. Lotz, "Platelet-derived growth factor-coated decellularized meniscus scaffold for integrative healing of meniscus tears," Acta Biomaterialia, vol. 76, pp. 126-134, 2018.

[77] M. B. Pabbruwe, E. Esfandiari, W. Kafienah, J. F. Tarlton, and A. P. Hollander, "Induction of cartilage integration by a chondrocyte/collagen-scaffold implant," Biomaterials, vol. 30, no. 26, pp. 4277-4286, 2009.

[78] N. A. Smyth, A. M. Haleem, C. D. Murawski, H. T. Do, J. T. Deland, and J. G. Kennedy, "The effect of platelet-rich plasma on autologous osteochondral transplantation: an In Vivo rabbit model," The Journal of Bone \& Joint Surgery, vol. 95, no. 24, pp. 2185-2193, 2013.
[79] H. Cho, J. Kim, S. Kim et al., "Dual delivery of stem cells and insulin-like growth factor-1 in coacervate-embedded composite hydrogels for enhanced cartilage regeneration in osteochondral defects," Journal of Controlled Release, vol. 327, pp. 284-295, 2020.

[80] B. Ren, X. Hu, J. Cheng et al., "Synthesis and characterization of polyphosphazene microspheres incorporating demineralized bone matrix scaffolds controlled release of growth factor for chondrogenesis applications," Oncotarget, vol. 8, no. 69, pp. 114314-114327, 2017.

[81] Z. Luo, L. Jiang, Y. Xu et al., "Mechano growth factor (MGF) and transforming growth factor (TGF)- $\beta 3$ functionalized silk scaffolds enhance articular hyaline cartilage regeneration in rabbit model," Biomaterials, vol. 52, pp. 463-475, 2015.

[82] J. Lu, X. Shen, X. Sun et al., "Increased recruitment of endogenous stem cells and chondrogenic differentiation by a composite scaffold containing bone marrow homing peptide for cartilage regeneration," Theranostics, vol. 8, no. 18, pp. 5039-5058, 2018.

[83] X. Lv, C. Sun, B. Hu et al., "Simultaneous recruitment of stem cells and chondrocytes induced by a functionalized selfassembling peptide hydrogel improves endogenous cartilage regeneration," Frontiers in Cell and Development Biology, vol. 8 , no. $864,2020$.

[84] X. Cui, K. Breitenkamp, M. G. Finn, M. Lotz, and D. D. D'Lima, "Direct human cartilage repair using threedimensional bioprinting technology," Tissue Engineering. Part A, vol. 18, no. 11-12, pp. 1304-1312, 2012.

[85] J. H. Shim, K.-M. Jang, S. K. Hahn et al., “Three-dimensional bioprinting of multilayered constructs containing human mesenchymal stromal cells for osteochondral tissue regeneration in the rabbit knee joint," Biofabrication, vol. 8, no. 1, p. 014102, 2016.

[86] Y. Yang, G. Yang, Y. Song, Y. Xu, S. Zhao, and W. Zhang, “3D bioprinted integrated osteochondral scaffold-mediated repair of articular cartilage defects in the rabbit knee," Journal of Medical and Biological Engineering, vol. 40, no. 1, pp. 7181, 2020.

[87] A. C. Daly and D. J. Kelly, "Biofabrication of spatially organised tissues by directing the growth of cellular spheroids within 3D printed polymeric microchambers," Biomaterials, vol. 197, pp. 194-206, 2019.

[88] L. Gong, J. Li, J. Zhang et al., "An interleukin-4-loaded bilayer 3D printed scaffold promotes osteochondral regeneration," Acta Biomaterialia, 2020.

[89] Y. Sun, Y. You, W. Jiang, B. Wang, Q. Wu, and K. Dai, “3D bioprinting dual-factor releasing and gradient-structured constructs ready to implant for anisotropic cartilage regeneration," Science Advances, vol. 6, no. 37, p. eaay1422, 2020.

[90] K. S. Lim, F. Abinzano, P. N. Bernal et al., "One-step Photoactivation of a dual-functionalized bioink as cell carrier and cartilage-binding glue for chondral regeneration," Advanced Healthcare Materials, vol. 9, no. 15, article e1901792, 2020.

[91] Y. Sun, Q. Wu, Y. Zhang, K. Dai, and Y. Wei, “3D-bioprinted gradient-structured scaffold generates anisotropic cartilage with vascularization by pore-size-dependent activation of HIF $1 \alpha / F A K$ signaling axis," Nanomedicine: Nanotechnology, Biology and Medicine, vol. 37, p. 102426, 2021.

[92] C. Di Bella, S. Duchi, C. D. O'Connell et al., "In situ handheld three-dimensional bioprinting for cartilage regeneration," Journal of Tissue Engineering and Regenerative Medicine, vol. 12, no. 3, pp. 611-621, 2018. 
[93] K. Ma, T. Zhao, L. Yang et al., "Application of roboticassisted in situ $3 \mathrm{D}$ printing in cartilage regeneration with HAMA hydrogel: an In Vivo study," Journal of Advanced Research, vol. 23, pp. 123-132, 2020.

[94] M. Yáñez-Mó, P. R. Siljander, Z. Andreu et al., "Biological properties of extracellular vesicles and their physiological functions," Journal of Extracellular Vesicles, vol. 4, no. 1, p. 27066, 2015.

[95] Y. Wang, D. Yu, Z. Liu et al., "Exosomes from embryonic mesenchymal stem cells alleviate osteoarthritis through balancing synthesis and degradation of cartilage extracellular matrix," Stem Cell Research \& Therapy, vol. 8, no. 1, pp. 113, 2017.

[96] G. Mao, Z. Zhang, S. Hu et al., "Exosomes derived from miR92a-3p-overexpressing human mesenchymal stem cells enhance chondrogenesis and suppress cartilage degradation via targeting WNT5A," Stem Cell Research \& Therapy, vol. 9, no. 1, pp. 247-247, 2018.

[97] S.-C. Tao, T. Yuan, Y. L. Zhang, W. J. Yin, S. C. Guo, and C. Q. Zhang, "Exosomes derived from miR-140-5p-overexpressing human synovial mesenchymal stem cells enhance cartilage tissue regeneration and prevent osteoarthritis of the knee in a rat model," Theranostics, vol. 7, no. 1, pp. 180-195, 2017.

[98] M. Zavatti, F. Beretti, F. Casciaro, E. Bertucci, and T. Maraldi, "Comparison of the therapeutic effect of amniotic fluid stem cells and their exosomes on monoiodoacetate-induced animal model of osteoarthritis," BioFactors, vol. 46, no. 1, pp. 106-117, 2020.

[99] C. Liu, Y. Li, Z. Yang, Z. Zhou, Z. Lou, and Q. Zhang, "Kartogenin enhances the therapeutic effect of bone marrow mesenchymal stem cells derived exosomes in cartilage repair," Nanomedicine, vol. 15, no. 3, pp. 273-288, 2020.

[100] S. Zhang, S. J. Chuah, R. C. Lai, J. H. Hui, S. K. Lim, and W. S. Toh, "MSC exosomes mediate cartilage repair by enhancing proliferation, attenuating apoptosis and modulating immune reactivity," Biomaterials, vol. 156, pp. 16-27, 2018.

[101] J. Li, Z. Ding, Y. Li et al., "BMSCs-derived exosomes ameliorate pain via abrogation of aberrant nerve invasion in subchondral bone in lumbar facet joint osteoarthritis," Journal of Orthopaedic Research, vol. 38, no. 3, pp. 670-679, 2020.

[102] J. Wu, L. Kuang, C. Chen et al., "miR-100-5p-abundant exosomes derived from infrapatellar fat pad MSCs protect articular cartilage and ameliorate gait abnormalities via inhibition of mTOR in osteoarthritis," Biomaterials, vol. 206, pp. 87$100,2019$.

[103] L. Yan, G. Liu, and X. Wu, “The umbilical cord mesenchymal stem cell-derived exosomal lncRNA H19 improves osteochondral activity through miR-29b-3p/FoxO3 axis," Clinical and Translational Medicine, vol. 11, no. 1, pp. e255-e255, 2021.

[104] L. Yan and X. Wu, "Exosomes produced from 3D cultures of umbilical cord mesenchymal stem cells in a hollow-fiber bioreactor show improved osteochondral regeneration activity," Cell Biology and Toxicology, vol. 36, no. 2, pp. 165-178, 2020.

[105] D. Sabry, A. Shamaa, M. Amer et al., "The effect of mesenchymal stem cell derived microvesicles in repair of femoral chondral defects in dogs," Journal of Musculoskeletal Research, vol. 21, no. 2, p. 1850006, 2018.

[106] M. Xie, W. Xiong, Z. She et al., "Immunoregulatory effects of stem cell-derived extracellular vesicles on immune cells," Frontiers in Immunology, vol. 11, 2020.
[107] S. Zhang, W. C. Chu, R. C. Lai, S. K. Lim, J. H. P. Hui, and W. S. Toh, "Exosomes derived from human embryonic mesenchymal stem cells promote osteochondral regeneration," Osteoarthritis and Cartilage, vol. 24, no. 12, pp. 2135-2140, 2016.

[108] Y. Jiang, X. Zhang, J. Wu, L. Qi, and K. Liu, "Hypoxic ADSCs-derived EVs Promote the Proliferation and Chondrogenic Differentiation of Cartilage Stem/progenitor Cells," in Adipocyte, no. 1, pp. 322-337, 2020.

[109] H. Hu, L. Dong, Z. Bu et al., "miR-23a-3p-abundant small extracellular vesicles released from Gelma/nanoclay hydrogel for cartilage regeneration," Journal of Extracellular Vesicles, vol. 9, no. 1, p. 1778883, 2020.

[110] C. H. Woo, H. K. Kim, G. Y. Jung et al., "Small extracellular vesicles from human adipose-derived stem cells attenuate cartilage degeneration," Journal of Extracellular Vesicles, vol. 9, no. 1, p. 1735249, 2020.

[111] Q. Liao, B. J. Li, Y. Li et al., "Low-intensity pulsed ultrasound promotes osteoarthritic cartilage regeneration by BMSCderived exosomes via modulating the NF- $\kappa \mathrm{B}$ signaling pathway," International Immunopharmacology, vol. 97, article 107824, 2021.

[112] L. A. Vonk, S. F. J. van Dooremalen, N. Liv et al., "Mesenchymal stromal/stem cell-derived extracellular vesicles promote human cartilage RegenerationIn Vitro," Theranostics, vol. 8, no. 4, pp. 906-920, 2018.

[113] R. Wang, W. Jiang, L. Zhang et al., "Intra-articular delivery of extracellular vesicles secreted by chondrogenic progenitor cells from MRL/MpJ superhealer mice enhances articular cartilage repair in a mouse injury model," Stem Cell Research \& Therapy, vol. 11, no. 1, pp. 1-14, 2020.

[114] Y. Kato and D. Gospodarowicz, "Sulfated proteoglycan synthesis by confluent cultures of rabbit costal chondrocytes grown in the presence of fibroblast growth factor," The Journal of Cell Biology, vol. 100, no. 2, pp. 477-485, 1985.

[115] I. Martin, G. Vunjak-Novakovic, J. Yang, R. Langer, and L. E. Freed, "Mammalian chondrocytes expanded in the presence of fibroblast growth factor 2 maintain the ability to differentiate and regenerate three-dimensional cartilaginous tissue," Experimental Cell Research, vol. 253, no. 2, pp. 681-688, 1999.

[116] M. Jakob, O. Demarteau, D. Schäfer et al., "Specific growth factors during the expansion and redifferentiation of adult human articular chondrocytes enhance chondrogenesis and cartilaginous tissue formation In Vitro," Journal of Cellular Biochemistry, vol. 81, no. 2, pp. 368-377, 2001.

[117] N. Veilleux and M. Spector, "Effects of FGF-2 and IGF-1 on adult canine articular chondrocytes in type II collagen-glycosaminoglycan scaffolds In Vitro," Osteoarthritis and Cartilage, vol. 13, no. 4, pp. 278-286, 2005.

[118] R. L. Sah, A. C. Chen, A. J. Grodzinsky, and S. B. Trippel, "Differential effects of bFGF and IGF-I on matrix metabolism in calf and adult bovine cartilage explants," Archives of Biochemistry and Biophysics, vol. 308, no. 1, pp. 137-147, 1994.

[119] Y. Chiyokura, "Effects of IGF-I, IGF-II, and PDGF on the proliferation of rabbit articular chondrocytes in culture," Journal of Orthopaedic Science, vol. 1, no. 1, pp. 59-63, 1996.

[120] P. J. Verschure, C. J. F. Van Noorden, J. Van Marle, and W. B. Van Den Berg, "Articular cartilage destruction in experimental inflammatory arthritis: insulin-like growth factor-1 regulation of proteoglycan metabolism in chrondrocytes," The Histochemical Journal, vol. 28, no. 12, pp. 835-857, 1996. 
[121] L. A. Fortier, G. Lust, H. O. Mohammed, and A. J. Nixon, "Coordinate upregulation of cartilage matrix synthesis in fibrin cultures supplemented with exogenous insulin-like growth factor-I," Journal of Orthopaedic Research, vol. 17, no. 4, pp. 467-474, 1999.

[122] A. J. Nixon, L. A. Fortier, J. Williams, and H. Mohammed, "Enhanced repair of extensive articular defects by insulinlike growth factor-I-laden fibrin composites," Journal of Orthopaedic Research, vol. 17, no. 4, pp. 475-487, 1999.

[123] M. K. Boushell, C. Z. Mosher, G. K. Suri et al., "Polymeric mesh and insulin-like growth factor 1 delivery enhance cell homing and graft-cartilage integration," Annals of the New York Academy of Sciences, vol. 1442, no. 1, pp. 138-152, 2019.

[124] A. Gigout, H. Guehring, D. Froemel et al., "Sprifermin (rhFGF18) enables proliferation of chondrocytes producing a hyaline cartilage matrix," Osteoarthritis and Cartilage, vol. 25, no. 11, pp. 1858-1867, 2017.

[125] M. L. Sennett, G. R. Meloni, A. J. E. Farran, H. Guehring, R. L. Mauck, and G. R. Dodge, "Sprifermin treatment enhances cartilage integration in an In Vitro repair model," Journal of Orthopaedic Research, vol. 36, no. 10, pp. 2648-2656, 2018.

[126] Y. Hong, N. Liu, R. Zhou et al., "Combination therapy using Kartogenin-based Chondrogenesis and complex polymer scaffold for cartilage defect regeneration," ACS Biomaterials Science \& Engineering, vol. 6, no. 11, pp. 6276-6284, 2020.

[127] H. Diao, J. Wang, C. Shen et al., "Improved cartilage regeneration utilizing mesenchymal stem cells in TGF-betal geneactivated scaffolds," Tissue Engineering. Part A, vol. 15, no. 9, pp. 2687-2698, 2009.

[128] A. R. A. Szojka, B. D. Lyons, C. N. Moore et al., "Hypoxia and TGF- $\beta 3$ synergistically mediate inner meniscus-like matrix formation by fibrochondrocytes," Tissue Engineering. Part A, vol. 25, no. 5-6, pp. 446-456, 2019.

[129] Z. Jia, S. Wang, Y. Liang, and Q. Liu, “Combination of kartogenin and transforming growth factor- $\beta 3$ supports synovial fluid-derived mesenchymal stem cell-based cartilage regeneration," American Journal of Translational Research, vol. 11, no. 4, pp. 2056-2069, 2019.

[130] V. Jeyakumar, E. Niculescu-Morzsa, C. Bauer, Z. Lacza, and S. Nehrer, "Platelet-rich plasma supports proliferation and redifferentiation of chondrocytes during in vitro expansion," Frontiers in Bioengineering and Biotechnology, vol. 5, no. 75, 2017.

[131] C. Sermer, R. Kandel, J. Anderson, M. Hurtig, and J. S. Theodoropoulos, "The role of platelet-rich plasma in promoting cartilage integration and chondrocyte Migration," The Journal of Sports Medicine, vol. 5, 7_suppl6, p. 2325967117S0022, 2017. 